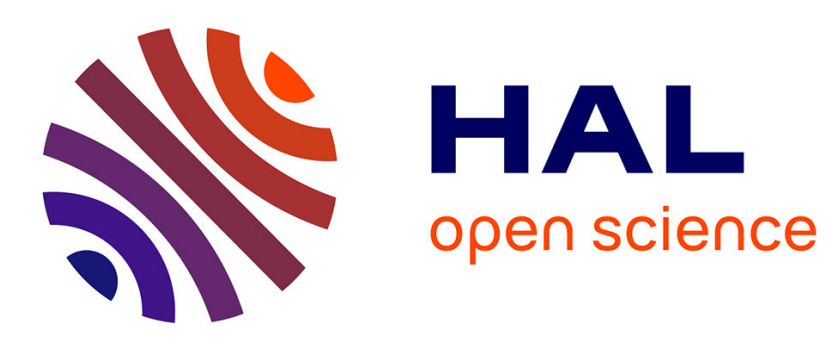

\title{
Domestiques d'ici et d'ailleurs
}

Félicie Drouilleau, Agnès Fine, Mélanie Jacquemin, Isabelle Puech

\section{To cite this version:}

Félicie Drouilleau, Agnès Fine, Mélanie Jacquemin, Isabelle Puech. Domestiques d'ici et d'ailleurs.

Travail, genre et sociétés, 2009, N²2 (2), pp.25. 10.3917/tgs.022.0025 . halshs-02488896

\section{HAL Id: halshs-02488896 \\ https://shs.hal.science/halshs-02488896}

Submitted on 1 Apr 2020

HAL is a multi-disciplinary open access archive for the deposit and dissemination of scientific research documents, whether they are published or not. The documents may come from teaching and research institutions in France or abroad, or from public or private research centers.
L'archive ouverte pluridisciplinaire HAL, est destinée au dépôt et à la diffusion de documents scientifiques de niveau recherche, publiés ou non, émanant des établissements d'enseignement et de recherche français ou étrangers, des laboratoires publics ou privés. 


\section{Domestiques d'ici et d'ailleurs}

Présentation du dossier, Travail, genre et sociétés, 22(2), n², 2009.

Nées dans le sillage des Women's Studies à la fin des années 70, les recherches en sciences humaines et sociales sur l'emploi domestique sont fortement redevables des réflexions féministes sur la valeur économique de l'activité des femmes dans le foyer. La remise en question d'une certaine improductivité féminine fait très vite place à un nécessaire examen de la condition de "bonne à tout faire ». Les historiens, anglo-saxons puis français, seront les premiers à se pencher sur sujet ${ }^{1}$. Des travaux en économie, sociologie et anthropologie suivent de peu ce mouvement, démontrant alors la pertinence d'une approche pluridisciplinaire de la question ancillaire ${ }^{2}$. Cependant, comme le soulignent Blandine Destremeau et Bruno Lautier en 2002, dans leur introduction à un numéro thématique sur la domesticité $^{3}$, si l'on considère que le « travail fait par les employées domestiques est une des principales formes d'emploi féminin dans la plus grande partie du monde », le nombre des études scientifiques qui lui ont été consacrées reste très faible. Les deux auteurs identifient les deux raisons essentielles de cette occultation : le travail domestique se déroule dans la sphère privée à l’intérieur des murs du domicile, d'une part et «il ne produit rien dans la comptabilité nationale » d'autre part ${ }^{4}$. En outre il ne constitue pas un problème politique majeur. On peut se référer à ce texte très synthétique qui, à la fois, met en évidence les caractères spécifiques de ce travail et la grande diversité des situations dont les «principaux

\footnotetext{
Voir les ouvrages de Theresa Mac Bride (1976, The domestic revolution: the modernisation of household service in England and France 1820-1920, New-York, Holmes and Meier publishers) ou encore de David Katzman (1978, Seven days a week : women and domestic service in industrializing America, New-York, Oxford University Press) pour l'Angleterre et les Etats-Unis, pays dans lesquels la production scientifique sur la domesticité reste soutenue. En France, si les essais de Geneviève Fraisse (1979, Femmes toutes mains: essai sur le service domestique, Paris, Editions du Seuil, rééd.2008), Anne Martin-Fugier (1979, La place des bonnes. La domesticité féminine à Paris en 1900, Paris, Editions Grasset et Fasquelle), Pierre Guiral et Guy Thuillier (1978, La vie quotidienne des domestiques en France au XIXe siècle, Paris, Hachette), et JeanPierre Gutton (1981, Domestiques et serviteurs dans la France de l'Ancien Régime, Paris, Aubier Montaigne) sont désormais des classiques en histoire sociale, ils seront peu suivis d'effets jusqu'aux années 2000, à de rares exceptions près. (Pour cette revue de la littérature, on pourra se reporter au livre de Valérie Piette : 2000, Domestiques et Servantes. Des vies sous conditions. Essai sur le travail domestique en Belgique au 19e siècle, Bruxelles, Académie Royale de Belgique.) Sur l'histoire de la domesticité en Italie, voir la bibliographie de Raffaella Sarfati, Vita di casa. Abitare, mangiare, vestire nell'Europa moderna, Rome/Bari, Laterza, 1999, ainsi que Angiolina Arru et Franco Ramella (dir), «L'Italia delle migrazioni interne : donne, uomini, mobilità in età moderna e contemporanea », Donzelli, 2003
}

2 On notera, pour l'Amérique du Nord, les travaux de Judith Rollins (1985, Between women. Domestics and their employers, Philadelphia, Temple University Press) et d'Evelyn Glenn Nakano (1986, Issei, Nisei, War Bride. Three generations of Japanese American women in domestic service, Philadelphia, Temple University Press). L'ouvrage d'Elsa Chaney et Mary Garcia Castro (1989, Muchachas no more, household workers in Latin America and the Caribbean, Philadelphia, Temple University Press) est maintenant une référence pour les études sur le service domestique en Amérique Latine. Enfin, dans les années 1980, des travaux pionniers émergent également en Afrique : Jacklyn Cock, 1980, Maids and Madams. A study in the politics of exploitation, Johannesburg, Ravan Press; Suzanne Gordon; 1985, A Talent for Tomorrow. Life and stories of South African servants, Johannesburg, Ravan Press; Karen Tranberg Hansen, 1989, Distant companions. Servants and employers in Zambia, 1900-1985, Ithaca/London, Cornell University Press. (Pour cette revue de littérature, voir K. T. Hansen, 1991, "Domestic service. What's in it for anthropology?”, Reviews in Anthropology, vol.16, pp. 47-62.)

3 Femmes en domesticité, les domestiques du Sud, au Nord et au Sud, Revue Tiers Monde, $n^{\circ} 170$, avril-juin 2002, p 249-264.

4 Sur la non-mesure du travail des femmes à la maison dans les comptes nationaux, voir les analyses des économistes J.K. Galbraith et Marilyn Waring, notamment diffusées dans le documentaire Who is counting ? Sexe, mensonges et mondialisation, Terre Nash, 1996. 
paramètres représentent autant de catégories analytiques : l'origine géographique (nationale, internationale), le statut résidentiel (dans la maison de la patronne ou ailleurs), la situation visà-vis du droit du travail ; l'existence d'une dette; le niveau de rémunération ; le type de tâches et le degré de disponibilités exigés; et d'une façon plus impalpable, l'ambiance de travail, l'amour, la haine, la confiance, la défiance. » (2002, p 254).

Mais, au tournant des années 2000, les études se multiplient et sur tous les continents, qui rendent compte de l'intensification des migrations internationales des pays du Sud vers les pays du Nord, ainsi que du rôle de l'emploi domestique dans ces migrations. La tendance est particulièrement marquée en Europe du Sud (Italie, Espagne) avec l'emploi de femmes originaires des Philippines, du Maghreb, d'Amérique Latine et des pays de l'Est ${ }^{5}$ pour faire le ménage et assurer la garde des personnes âgées de jour comme de nuit. L'Allemagne et les Pays-Bas semblent également concernés par le phénomène ${ }^{6}$, ainsi que la France et la Belgique $^{7}$. C'est en Amérique du Nord que les études les plus novatrices voient le jour. Plusieurs chercheuses ${ }^{8}$ popularisent ainsi la notion de "famille transnationale » (transnational families), «volet féminin de la globalisation, dans lequel des millions de [femmes] pauvres des pays du Sud migrent pour faire le "travail des femmes » du Nord travail qu'[elles] ne savent ou ne veulent plus faire. » (Ehrenreich et Russel Hochschild, 2002, p. 3) Ces migrantes ont souvent une famille à nourrir et des enfants à élever qu'elles laissent alors à la charge (care) de grands-mères, soeurs, ou belles-soeurs. La globalisation implique donc un partage international de l'affect et une " chaîne globale du soin » (global care chain) (Lutz, 2002, p. 100), dans laquelle les femmes des pays du Sud privent leurs enfants de soin et d'attention, pour donner leur affection aux enfants de leurs patrons. L'écho important, en Amérique du Nord, des théories féministes du care ${ }^{9}$ n'a sans doute pas été sans effet sur ce renouvellement des recherches sur le service domestique, dans sa modalité internationalisée, et son caractère proprement "moderne ". Le terme care pourrait se traduire en français par " sollicitude, soin, souci ». Mais, pour Patricia Paperman et Sandra Laugier, il désigne plus généralement l'ensemble des " phénomènes vus mais non remarqués assurant l'entretien [...] d'un monde humain » (2005, p. 10). L'éthique du care devient ainsi une éthique féministe du sensible, valorisant le "souci des autres ». Ces analyses définissent le service domestique comme un travail d'attention et de soins à l'autre, qui crée des relations continues entre les " care givers " et les "care receivers " - la qualité du travail effectué se mesurant alors à l'aune de ces relations ${ }^{10}$.

Francesca Scrinzi, 2003, «Les employées de maison migrantes en Italie. Ethnicisation et contrôle dans le circuit catholique à Gênes ", Canadian woman Studies/Les Cahiers de la Femme, vol. 22, n³/4, pp. 182187 ; Rossana Trifiletti, 2005, «Le soin aux personnes âgées et les parcours d'intégration des immigrés en Italie », Retraite et société, vol.1, n44, pp. 149-173 ; Laura Oso Casas, 2003, Domestiques, concierges et prostituées: migration et mobilité sociale des femmes immigrées espagnoles à Paris, équatoriennes et colombiennes en Espagne, Paris, Thèse de doctorat, Université Panthéon Sorbonne.

6 Helma Lutz, 2002, « At your service madam! The globalization of domestic service » in Feminist Review, n ${ }^{\circ} 70$, pp. 89-104.

7 Mozère Liane, 2001, "La Philippine ou la Mercédès Benz des domestiques. Entre archaïsmes et modernité", Sextant, n 15-16, pp. 297-318. ; Isabelle Puech. 2007, L'emploi des femmes dans les services à domicile. Quelle croissance, quelle qualité, quelles politiques. Une comparaison France-Belgique des femmes de ménage travaillant chez les particuliers, Rapport de recherche post-doctorale, ULB-DULBEA, 31 janvier.

8 Rhacel Salazar Parreñas, 2001, Servants of the globalization. Women, migration and domestic work, Stanford, Stanford University Press ; Barbara Ehrenreich, Arlie Russel Hoschschild, 2002, Global Woman, nannies, maids and sex-workers in the new economy, New-York, Holt.

9 Pour une introduction française aux théories du care, voir Patricia Paperman et Sandra Laugier, 2005, Le souci des autres. Ethique et politique du care, Paris, Editions de l'Ecole des Hautes Etudes en Sciences Sociales.

10 Pour les liens entre service domestique et étude sur le care, voir : Joan C. Tronto, 2002, « The "nanny" 
La « modernité » de l'emploi domestique n'est cependant pas réductible à son lien avec les migrations internationales. Phénomène ancien, le travail domestique des enfants et adolescent-es ressort par exemple comme une spécificité contemporaine de l'emploi domestique en de nombreux pays du Sud. La diversité des pratiques, les dimensions multiples et l'ampleur qu'il recouvre ont eu pour effet d'en faire une préoccupation nouvelle pour les organisations de protection de l'enfance, les associations et la littérature militante, mais aussi pour la recherche. Activité majeure des enfants et jeunes travailleurs de sexe féminin, il n'est encore que rarement perçu en regard de l'importance des migrations juvéniles féminines de travail - internes, transfrontalières voire internationales - qu'il sous-tend. Parce qu'il permet d'éclairer sous un jour nouveau des débats classiques et contemporains en sciences sociales, qu'il met en résonance l'ensemble des questions que soulève la domesticité aujourd'hui, ce champ mérite d'être davantage exploré, notamment autour de la question de la catégorisation de ces jeunes travailleuses, et de leur mise en visibilité pour une meilleure prise en compte et protection.

Qu'elles soient ou non mineures, on ne saurait oublier plus longtemps que la prise de parole des travailleuses domestiques est porteuse de transformations importantes. En Amérique Latine et en Afrique, le service domestique prend ainsi des formes nouvelles, liées aux évolutions économiques, politiques et législatives : le salariat du travail des enfants, l'impact du conflit armé, l'accès aux droits sociaux, comme le montrent respectivement Mélanie Jacquemin pour la Côte-d'Ivoire, Félicie Drouilleau pour la Colombie et Dominique Vidal pour le Brésil dans leurs contributions pour ce dossier.

Celui-ci est issu d'une journée d'étude, organisée à Toulouse en décembre 2007, qui a permis de croiser les regards, entre plusieurs disciplines et aires culturelles, pour entrevoir la diversité des dynamiques à l'œuvre sur le travail domestique féminin ${ }^{11}$. Manquait dans le débat un exemple concernant les services domestiques dans un pays développé. C'est pourquoi une contribution sur la situation française a été sollicitée par la suite. Le lecteur devra donc tenir compte de la grande diversité des contextes sociaux dont il est question ici, la France, la Côte d’Ivoire, la Colombie, et le Brésil et de la variété des approches disciplinaires et méthodologiques.

Claire Marbot s’interroge en statisticienne sur les caractéristiques des ménages qui, en France, ont recours aux services à domicile. C'est donc le profil des employeurs qu'elle cherche à définir, ou plutôt des employeuses. En effet, l'auteure met en évidence le rôle déterminant du statut socioprofessionnel des femmes des couples observés : elles sont actives, mères d'enfants en bas âge, elles ont un niveau de vie relativement élevé, mais surtout elles sont fortement diplômées et exercent elles-mêmes une profession qualifiée.

Le texte de Mélanie Jacquemin sur les formes de la domesticité féminine enfantine et juvénile à Abidjan nous entraîne dans un contexte en tous points différents. Le caractère massif du travail extorqué aux filles par leur " tantie », par leur tutrice ou leur employeuse, dans le cadre de relations de parenté ou de voisinage ou en dehors, dans un rapport de

question in feminism », Hypatia, vol. 17, n², pp. 34-51. L'ouvrage dirigé par Luz Gabriela Arango et Pascale Molinier (2009, à paraître), Etica y trabajo del cuidado, Bogota, Universidad Nacional de Colombia) comprend également une section dédiée aux emplois domestiques.

11 La journée d'études a été organisée à Toulouse dans le cadre du séminaire Genre de l'Ecole doctorale TESC par Félicie Drouilleau et Carmen Sandoval, avec l'appui d'Agnès Fine et d'Angelina Peralva, et le soutien financier de plusieurs institutions dont la MSHT, le PPF Genre de l'EHESS, le PPF genre Arpège, le Centre d'anthropologie sociale et le CERS (LISST). Valérie Piette a présenté son travail sur les domestiques en Belgique au XIXe siècle et Laura Oso Casas les résultats de sa thèse de doctorat portant sur l'immigration de femmes colombiennes et équatoriennes en Espagne, dans le service domestique et la prostitution. 
salariat, est la condition même de la survie des femmes qui les emploient et de leurs familles. Ici, dans un contexte de faible industrialisation et de grave crise économique, c'est la fonction économique essentielle de ce travail féminin enfantin et juvénile, au prix d'une surexploitation importante des filles, qui est mis en évidence,. L'auteure note cependant une évolution récente vers le salariat, ce qui a pour effet de donner une valeur marchande à ce travail et partant, du prix à celle qui l'accomplit.

Félicie Drouilleau, étudiant l'évolution du travail domestique féminin à Bogotà dans les quatre dernières décennies, analyse l'impact spécifique du conflit armé colombien sur la demande d'emploi dans ce secteur et les transformations du profil des femmes qui l'accomplissent. Ce ne sont plus de petites filles ou jeunes femmes du monde rural qui sont placées par leurs parents à la ville dans la parenté ou auprès de personnes à qui on les recommande, mais des mères de familles, privées de maris et chargées d'enfants, qui fuyant la violence, arrivent en masse à Bogotà. Là, en raison de la discrimination dont sont l'objet les personnes déplacées par les violences, elles ont toutes les difficultés du monde à entrer dans une maison pour y faire un des seuls emplois qui leur soit ouvert et qu'elles souhaitent exercer, celui de domestique.

Face à l'exploitation et à la misère vécue par la majorité des domestiques à Abidjan ou à Bogotà, la situation des Brésiliennes contraste de manière positive. Elles ont en effet obtenu des droits sociaux importants depuis l'instauration de la démocratie en 1988. Dominique Vidal analyse les effets de ces acquis sur la représentation que les travailleuses domestiques se font d'elles-mêmes et de leurs relations de travail. Même si les droits sont insuffisants ou mal appliqués, ces changements juridiques ont une portée économique, politique et symbolique immense.

Ces quatre contributions nous montrent quelques unes des mutations silencieuses du service domestique en Europe, en Afrique et en Amérique Latine. A côté de transformations plus spectaculaires, comme celles liées aux migrations internationales ou au phénomène de traite des femmes et des enfants, cet emploi continue d'évoluer et de s'adapter aux changements sociaux, économiques et politiques, témoignant d'une vivacité qui est loin d'en faire une activité des temps passés.

Félicie Drouilleau, Agnès Fine, Mélanie Jacquemin, Isabelle Puech 